\title{
Morphology, Structure, and Enhanced Intramolecular Conduction in Ultralong Conjugated Polymer Brushes
}

\author{
Ian A. VonWald, ${ }^{\dagger}$ Mark M. Moog, ${ }^{\ddagger}$ Travis W. LaJoie, ${ }^{\dagger}$ Joshua D. Yablonski, ${ }^{\dagger}$ Dean M. DeLongchamp, ${ }^{\S}$ \\ Jason Locklin, ${ }^{\| \odot}$ Frank Tsui, ${ }^{*}+$ and Wei You* ${ }^{*}+\infty$ \\ ${ }^{\dagger}$ Department of Chemistry, University of North Carolina at Chapel Hill, Chapel Hill, North Carolina 27599, United States \\ ${ }^{\ddagger}$ Department of Physics and Astronomy, University of North Carolina at Chapel Hill, Chapel Hill, North Carolina 27599, United \\ States \\ ${ }^{\S}$ Materials Science and Engineering Division, National Institute of Standards and Technology, Gaithersburg, Maryland 20899, United \\ States \\ "Department of Chemistry and College of Engineering, University of Georgia, Athens, Georgia 30602, United States
}

\begin{abstract}
The morphological, structural, and electrical properties of poly(3-methylthiophene) polymer brush films grown on indium tin oxide up to $120 \mathrm{~nm}$ thick are reported. Optical spectroscopy experiments indicate that the average orientation of polymer chains is nearly isotropic at thicknesses below $10 \mathrm{~nm}$ and above $30 \mathrm{~nm}$, and exhibits mild vertical anisotropy in between. Atomic force microscopy shows that the film surfaces consist of column-shaped domains with an average cross-sectional area $\left(2.3 \times 10^{-3} \mu \mathrm{m}^{2}\right)$ and density $\left(200 \mu \mathrm{m}^{-2}\right)$ that are independent of film thickness. Analysis of the contact between printed Au electrodes and the polymer brush film shows that a small fraction of the film surface (i.e., the tallest columns) makes contact with the electrodes. The measured bulk resistivity
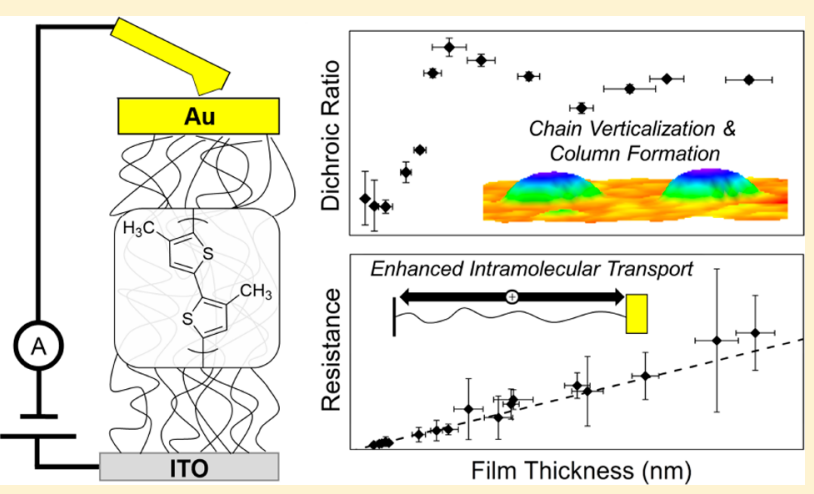
along the columns is $1.4 \times 10^{5} \Omega \cdot \mathrm{cm}, 2$ orders of magnitude lower than typical values for spuncast poly(3-alkylthiophene) films, while the resistance along individual polymer chains in the columns is estimated to be $360 \mathrm{G} \Omega / \mathrm{nm}$ per molecule, comparable to molecular wires that exhibit charge transport by intramolecular processes. The enhanced conduction is likely due to additional intramolecular transport pathways enabled by the electrode-polymer brush-electrode device architecture, establishing conjugated polymer brushes as a platform for studying the interplay among synthesis, morphology, and charge transport phenomena.
\end{abstract}

\section{INTRODUCTION}

Electrical conduction through polymers takes place via two primary types of pathways: through intermolecular $\pi$ stacks and along individual conjugated molecular chains. ${ }^{1-3}$ The mobility for intramolecular charge transport is expected to be much higher than that for the intermolecular counterpart, as shown in recent experimental ${ }^{4-6}$ and theoretical ${ }^{7}$ reports. Thus, it is a widely accepted notion and practice that intramolecular transport should be utilized in order to produce high efficiency organic semiconductor devices, such as solar cells, ${ }^{8-10}$ lightemitting diodes, ${ }^{11-13}$ transistors, ${ }^{3,14,15}$ and spin valves. ${ }^{16}$ Despite these efforts, major obstacles continue to limit our understanding of intramolecular processes. Specifically, individual polymer chains in bulk films (e.g., deposited by spincasting) do not contact both electrodes owing to structural disorder and large device thicknesses (typically $>100 \mathrm{~nm}$ ). Therefore, intermolecular and intramolecular conduction processes are necessarily convoluted in these films. ${ }^{6}$ For example, reports focused on improving chain alignment in bulk films using various techniques, such as nanopatterning, ${ }^{4}$ strain-induced alignment, ${ }^{5}$ and end-group fluorination, ${ }^{17}$ have led to varying degrees of enhancement in the bulk charge mobility, but they do not directly measure conduction along individual molecules. In contrast, self-assembled monolayers (SAMs) have been used successfully to study tunneling and intramolecular hopping transport through short molecular wires of lengths up to $20 \mathrm{~nm}$ (typically $<10 \mathrm{~nm}$ ). ${ }^{18-21}$ Covalent linkage of these molecular wires to one or both electrodes enables the characterization of intramolecular transport through individual and small groups of molecules, but this strategy is not scalable to longer molecular lengths due to synthetic (rigorous stepwise chemistry) and technical (low or negligible current in single or few molecule devices) limitations. Because of these challenges, intramolecular charge transport through

Received: January 2, 2018

Revised: $\quad$ March 27, 2018

Published: March 27, 2018 
long molecular wires $(>20 \mathrm{~nm})$ has not been studied experimentally.

Conjugated polymer brushes (CPBs) consisting of parallel molecular wires have the potential to be a well-suited materials platform for studying intramolecular charge transport. Like SAMs, CPBs are grafted to the bottom electrode but can be easily grown much longer than SAMs. Because of the covalent bond between polymer chains and the substrate, polymer chains contacting the top electrode in a vertical device may enable intramolecular charge transport processes to and from the bottom electrode. This electrode- $\mathrm{CPB}$-electrode device geometry makes it possible to directly probe the electrical resistivity of individual polymer chains (i.e., molecular resistivity) at the limit of negligible intermolecular charge transport processes. Previous reports on $\mathrm{CPBs}$, such as those composed of poly(3-methylthiophene) (P3MT), primarily focus on the procedure and kinetics of polymerization, ${ }^{22-26}$ and while these films were utilized in various applications including thermoelectronics, ${ }^{27}$ spintronics, ${ }^{28}$ and solar cells, ${ }^{29}$ their basic materials properties still remain largely unknown. ${ }^{30}$

In this article, we report a systematic study of the morphology, structure, and electronic properties of P3MT CPB films grown on indium tin oxide (ITO) surfaces as a function of film thickness, using primarily optical spectroscopy and noncontact atomic force microscopy (AFM). Au electrodes were printed on top of the $\mathrm{CPB}$ films using kinetic transfer printing $(\mathrm{KTP})^{31}$ to produce electrode-CPB-electrode devices. Our findings on the surface structure and morphology are summarized in a growth model for the P3MT CPBs and provide the critical means to analyze the polymer-electrode contact and charge transport effects through the P3MT CPB devices, leading to resistivity values for both the bulk films and individual polymer chains.

\section{EXPERIMENTAL SECTION}

Materials. All chemicals, reagents, and solvents were purchased from commercial sources (Sigma-Aldrich, Acros, VWR, etc.) and used without further purification unless otherwise noted. Dry THF was purified by distillation, and dry toluene (Fisher Chemicals) was used without further purification. Indium tin oxide (ITO) slides ( 1 in. $\times 1$ in., 145 $\mathrm{nm}$ sputtered ITO, and resistivity $20 \Omega / \mathrm{sq})$ and Si wafers (300 $\mathrm{nm}$ wet thermal oxide) were purchased from Thin Film Devices, Inc. and University Wafer, respectively. Au targets for sputtering were purchased from Kurt J. Lesker. 2-Bromo-3methyl-5-iodothiophene, 2-ferrocenyl-5-bromothiophene, (4bromobenzyl)phosphonic acid, and magnesiated 2-bromo-3methyl-5-iodothiophene and 2-ferrocenyl-5-bromothiophene were synthesized using established procedures. ${ }^{2,23,26}$

Preparation of ITO Substrates. ITO slides were sonicated in $18 \mathrm{M} \Omega$ deionized water, acetone, and isopropanol for $15 \mathrm{~min}$ each, then placed in RCA cleaning solution (5:1:1 $\mathrm{H}_{2} \mathrm{O}: \mathrm{H}_{2} \mathrm{O}_{2}: \mathrm{NH}_{4} \mathrm{OH}$ ) for an hour. After rinsing with water and isopropanol and drying under a stream of $\mathrm{N}_{2}$, the slides were cleaned with UV/ozone (model 42A, Jelight Company Inc.) for $15 \mathrm{~min}$. They were then immersed in an 8-slot staining dish with $30 \mathrm{~mL}$ of absolute ethanol containing (4bromobenzyl)phosphonic acid $(75 \mathrm{mg}, 0.3 \mathrm{mmol}$ ) overnight. They were quickly dried under a stream of $\mathrm{N}_{2}$ and heated in a glovebox at $150{ }^{\circ} \mathrm{C}$ overnight. Lastly, the slides were cleaned with water and ethanol and dried under $\mathrm{N}_{2}$ to yield monolayerfunctionalized ITO.
Surface Initiated Kumada Catalyst Transfer Polycondensation (SI-KCTP). SI-KCTP was adapted from a previous report. ${ }^{22} \mathrm{Pd}$-catalyzed SI-KCTP was chosen over Ni to reduce disproportionation and because polymer brushes grown using a $\mathrm{Ni}$ catalyst require prohibitively insulating monolayers to achieve vertical anisotropy. ${ }^{27}$ Monolayer-functionalized ITO slides were initially placed in a staining dish containing a 10 $\mathrm{mM}$ solution of $\mathrm{Pd}\left(\mathrm{P}^{t} \mathrm{Bu}_{3}\right)_{2}$ in dry toluene at $70^{\circ} \mathrm{C}$ for $3 \mathrm{~h}$ in a glovebox without stirring. The slides were then washed with excess toluene and THF, then immersed in a $0.15 \mathrm{mM}$ solution of the magnesiated 3-methylthiophene monomer in dry THF at $40{ }^{\circ} \mathrm{C}$ without stirring. At the desired time intervals (longer growth time corresponding to thicker films), slides were removed from the glovebox and sonicated briefly in chloroform, water, and isopropanol. The resulting P3MT CPB films were stored in the dark under inert atmosphere.

Cyclic Voltammetry (CV). Films for CV measurements of Pd initiator density were prepared similarly to those used to grow conjugated polymer brush films. After oxidative insertion of $\mathrm{Pd}\left(\mathrm{P}^{t} \mathrm{Bu}_{3}\right)_{2}$ into monolayer-functionalized ITO slides, the samples were placed in a $0.02 \mathrm{M}$ solution of magnesiated 2ferrocenyl-5-bromothiophene (prepared identically to the 3methylthiophene monomer for SI-KCTP) overnight without stirring, then sonicated in chloroform, water, and isopropanol to yield ferrocene-capped monolayers with a density equal to the Pd initiator density. CV was performed in a custom 3electrode electrochemical cell with the ITO substrate of the ferrocene-modified films acting as the working electrode, a platinum wire counter electrode, and $\mathrm{Ag} / \mathrm{AgCl}$ reference electrode. $^{26}$ Tetra(n-butylammonium hexafluorophosphate) was the electrolyte $(0.1 \mathrm{M})$ in thoroughly deoxygenated dichloromethane. Scans of ferrocene-capped monolayers on ITO were performed using a BASi Epsilon potentiostat, scanning from 0 to $+1.2 \mathrm{~V}$ at $100 \mathrm{mV} / \mathrm{s}$ with several scans to allow for equilibration. The integrated oxidation peak was then used to calculate the surface density of the capped monolayer.

UV-Vis and Polarized UV-Vis Measurements and Analysis. Normal incidence UV-visible absorption spectra of P3MT films were measured using a Shimadzu UV-2401PC spectrophotometer. Polarized variable angle UV-vis spectra were measured using a Thorlabs CCS200 spectrometer. Unpolarized light from a UV-vis/NIR lightsource (DH2000-BAL, Ocean Optics) was sent through a polarizing prism (GT5-A, Thor Laboratories) in either the vertically $(p)$ or horizontally $(s)$ aligned direction, while the sample mount was rotated $60^{\circ}$ with respect to incident light (inset, Figure 2a). Prior to the polarized measurements for each film, an unpolarized reference spectrum of each film was measured at normal incidence. Absorbance was calculated as $\log \left(T / T_{0}\right)$, where $T$ and $T_{0}$ are the transmission intensities at a given angle through an ITO substrate with or without a P3MT CPB film on top (i.e., sample or blank), respectively.

Grazing Incidence Wide Angle X-ray Scattering (GIWAXS). GIWAXS experiments were conducted at beamline 7.3.3 at the Advanced Light Source at Lawrence Berkeley National Laboratory. The collimated $10 \mathrm{keV}$ beam was approximately $300 \mu \mathrm{m}$ high and $800 \mu \mathrm{m}$ wide. Its angle of incidence from the surface plane was $0.14^{\circ}$, penetrating the grafted film and experiencing total internal reflection at the glass substrate. A $2 \mathrm{M}$ pixel Pilatus detector was used at a distance of $262 \mathrm{~mm}$, calibrated by the use of a silver behenate standard. Data were reduced using the Nika software package. ${ }^{32}$ The $q$ values were converted to $d$ values using $d=2 \pi / q$. 
Atomic Force Microscopy (AFM) and Scanning Electron Microscopy (SEM). AFM measurements were performed using an Asylum Atomic Force microscope (Asylum MFP-3D, Asylum Research). Tapping-mode topography images were taken using silicon cantilevers (Tap300Al-G, BudgetSensors) with a force constant of $40 \mathrm{~N} / \mathrm{m}$ and a resonance frequency of $300 \mathrm{kHz}$. To determine the nominal film thickness, P3MT films were scratched using a 20 gauge steel needle, and the scratched step was imaged using AFM. The film thickness is defined as the peak-to-peak height difference between the respective height histograms of the ITO surface and CPB film surface (Figure S1). SEM measurements were performed using a Hitachi S-4700 Field Emission SEM (Tokyo, Japan).

Preparation of Kinetic Transfer Printing (KTP) Donor Substrates. Silicon wafers (with a $300 \mathrm{~nm}$ surface oxide layer) were cut into roughly 2 in. $\times 1$ in. rectangles and placed in RCA cleaning solution $\left(2: 1: 1 \mathrm{H}_{2} \mathrm{O}: \mathrm{H}_{2} \mathrm{O}_{2}: \mathrm{NH}_{4} \mathrm{OH}\right)$ for an hour, then rinsed with water and ethanol, and dried under a stream of $\mathrm{N}_{2}$. The wafers were then cleaned with UV/Ozone for $15 \mathrm{~min}$, and then neat hexamethyldisilazane was applied to the surface of substrates. Excess hexamethyldisilazane was removed under a stream of $\mathrm{N}_{2}$. An array of $12.5 \mu \mathrm{m}$ diameter circular disks was lithographically patterned on the surface of the wafers using a negative photoresist (Ma-N 1410, thickness $\sim 1.0 \mu \mathrm{m}$, Micro Resist Technology) and photomask. A $200 \mathrm{~nm}$ thick film of $\mathrm{Au}$ was sputter deposited onto the pattern, followed by lift-off in acetone. Finally, the substrates were briefly etched $(2-3 \mathrm{~s})$ in $5 \% \mathrm{HF}$ to release features with a diameter of $12.5 \mu \mathrm{m}$ (measured using an optical microscope and AFM) from the oxide substrate.

KTP Process. This procedure was used to produce electrode-CPB-electrode devices for electrical measurements. A cross-linked poly(dimethylsiloxane) (PDMS) stamp (mixed 3.5:1 by weight with cross-linker, approximately $1 \mathrm{~cm} \times 1 \mathrm{~cm}$, Sylgard 184 Elastomer Kit, Dow Corning) was brought into conformal contact with the donor substrate. The stamp was quickly removed from the surface of the donor using pressure applied with a glass slide (rate of removal $>10 \mathrm{~cm} / \mathrm{s}$ ), transferring the $\mathrm{Au}$ features onto the stamp. The "inked" stamp was brought into conformal contact with the receiving substrate (e.g., the CPB film) with approximately $1 \mathrm{~N}$ of applied force and removed very slowly $(\sim 0.5 \mathrm{~mm} / \mathrm{s})$, transferring the $\mathrm{Au}$ features onto the receiving substrate. The PDMS stamp was cleaned to remove particulates between each transfer using scotch tape.

Conductive AFM (CAFM) Measurements. Two-terminal current-voltage $(I-V)$ measurements of the P3MT CPB devices fabricated by KTP were performed using the same Asylum AFM system with modified cantilevers of the same model as the tapping mode AFM measurements. The cAFM cantilevers were modified by sputtering alternating layers of $\mathrm{Cr}$ and $\mathrm{Au}$ (to improve adhesion) using the recipe $2.5 \mathrm{~nm} \mathrm{Cr}, 5.0$ $\mathrm{nm} \mathrm{Au}, 2.5 \mathrm{~nm} \mathrm{Cr}, 10.0 \mathrm{~nm} \mathrm{Au}, 2.5 \mathrm{~nm} \mathrm{Cr}, 35.0 \mathrm{~nm} \mathrm{Au}, 2.5 \mathrm{~nm}$ $\mathrm{Cr}$, and $50.0 \mathrm{~nm} \mathrm{Au}$, producing conductive tips of radii of $\sim 30$ $\mathrm{nm} .{ }^{33}$ Conductive measurements were performed in contact mode by applying a minimum force to make electrical contact to the top $\mathrm{Au}$ electrode $(<100 \mathrm{nN})$. The voltage bias was applied across the CPB device between the ITO substrate (positive bias) and the top Au electrode. Voltages were typically swept between $-1 \mathrm{~V}$ and $+1 \mathrm{~V}$ over $10 \mathrm{~s}$. For each CPB film, at least 20 devices were measured. From the $I-V$ measurements, the zero-bias resistance value was obtained from the inverse of the $I-V$ curve slope at zero voltage bias.

\section{RESULTS AND DISCUSSION}

P3MT CPB films were grown on ITO surfaces by surface initiated Kumada catalyst-transfer polycondensation (SIKCTP), using a phosphonic acid monolayer as the surface anchoring group (Figure 1a), as reported previously. ${ }^{22} \mathrm{~A}$
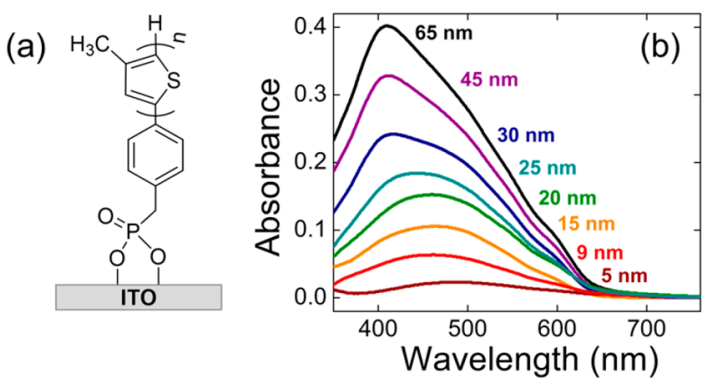

Figure 1. (a) Molecular structure of P3MT CPBs grown from ITO. (b) Normal incidence absorbance spectra of P3MT CPB films of various thicknesses.

catalyst initiator density of $1.3 \mathrm{~nm}^{-2}$ was determined by cyclic voltammetry $(\mathrm{CV})$ measurements prior to polymerization (Figure S2), similar to previous results. ${ }^{26}$ However, solution polymerization of P3MT was observed during SI-KCTP (evidenced by a solution color change from yellow to red and eventual precipitation of insoluble P3MT oligomers over time), indicating some desorption or chain transfer of the catalyst to monomer in solution, and a corresponding reduction in surface catalyst density during $\mathrm{CPB}$ growth.

Spectroscopic Analysis of Conjugated Polymer Brush Film Morphology and Structure. Normal incidence UV-vis spectroscopy experiments of the CPB films revealed two features at $\sim 610$ and $490 \mathrm{~nm}$ in all films, and a third feature at $415 \mathrm{~nm}$ in films $\geq 30 \mathrm{~nm}$ thick (Figure 1b). Optical anisotropy in these films was investigated using polarized UV-vis measurements at a $60^{\circ}$ incident angle (Figure 2). At this angle, the $p$ polarization couples strongly to the transition dipole along the polymer backbone of the vertically oriented polymer chains, ${ }^{34}$ while the $s$ polarization couples primarily to the transition dipole of horizontally oriented polymer chains. Thus, the dichroic ratio, $D R=A_{\mathrm{p}} / A_{\mathrm{s}}$, where $A_{p}$ and $A_{s}$ are the maximum intensity of absorption in the $p$ or $s$ polarization, respectively, was determined and used to compare the ensemble average orientation of the polymer chains in $\mathrm{CPB}$ films as they grow (average orientation given by the tilt angle $\theta$ in Figure 2a inset). A higher $D R$ corresponds to more vertical orientation, i.e., a lower $\theta$. The film thickness dependent values of $D R$ at oblique incidence exhibit three distinct regimes (Figure 2a): (i) a value of $\sim 0.6$ for thickness $<10 \mathrm{~nm}$, (ii) an increase in the $D R$ between 10 and $30 \mathrm{~nm}$ to a maximum of $\sim 1.2$, and (iii) a decrease in the $D R$ in films $>30 \mathrm{~nm}$ thick to a value slightly greater than 1 . Note that the $D R$ for the P3MT $\mathrm{CPB}$ films at normal incidence is 1 due to the isotropic nature of the films in the plane of the substrate (Figure S3). In addition to peak intensity, the peak position $\lambda_{\max }$ of the oblique incidence absorption spectra also have a polarization and thickness dependence. While the polarized spectra in thin P3MT CPB films $(<10 \mathrm{~nm})$ do not exhibit any difference in $\lambda_{\max }$ of the polarized spectra (Figure 2b), the $p$ polarized 

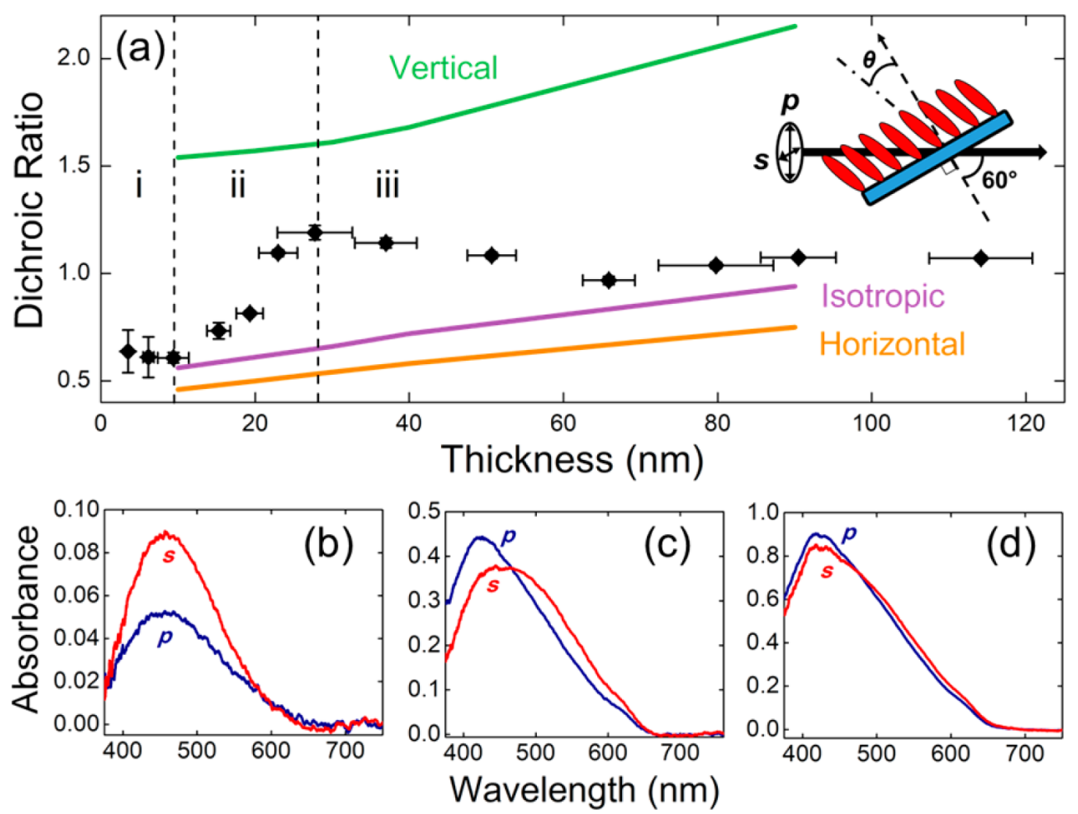

Figure 2. (a) Dichroic ratio for P3MT CPB films of various thicknesses, taken at $60^{\circ}$ incidence, with distinct growth regimes as labeled. Also shown are modeled spectra for nominally horizontal (orange), isotropic (purple), and vertical (green) orientations. Inset: experimental geometry for the polarized UV-vis measurements at an incident angle of $60^{\circ} . \theta$ denotes the tilt angle of monomers from the surface normal. (b-d) Polarized absorbance spectra of 10, 30, and $90 \mathrm{~nm}$ thick P3MT CPB films, respectively, at $60^{\circ}$ incidence.

spectrum exhibits a blue shift compared to the $s$ polarized spectrum as film thickness approaches $30 \mathrm{~nm}$ (Figure 2c). In much thicker films, both polarization spectra are blue-shifted (Figure 2d).

Grazing incidence wide-angle X-ray scattering (GIWAXS) measurements on P3MT CPB films of 30 and $120 \mathrm{~nm}$ thickness detected polycrystalline diffraction arcs at reciprocal space vectors $q=1.2$ and $1.8 \AA^{-1}$, while the measurement of thinner films yielded negligible diffraction intensities (Figure S4). The two arcs can be assigned to the (020) and (110) reflections, respectively, based on a crystal structure with staggered sheets $(\pi-\pi$ and lamellar lattice spacing of 5.1 and $7.5 \AA$, respectively). ${ }^{35}$ The two lattice vectors are orthogonal to the chain axis and exhibit a nearly isotropic distribution with a small amount of horizontal texture (Figures S5 and S6).

The absorption spectra of semicrystalline poly(alkylthiophene)s (P3ATs) have been studied extensively and are well understood in terms of an "H-J" model. ${ }^{36,37}$ The change in absorption spectra corresponding to local order convolves two effects: formation of intrachain J-aggregates due to increasing order and conjugation length in crystalline regions and interchain $\mathrm{H}$-aggregates formed by lamella packing of the extended chains. The extended dipole of the long conjugation segments gives rise to the somewhat unexpected result that the $\mathrm{H}$-aggregate coupling (interchain exciton bandwidth) decreases as the local order (conjugation length) increases. ${ }^{38}$ The resulting "weak $\mathrm{H}$-aggregate" well describes the absorption behavior of the vibronic series beginning at $\sim 600 \mathrm{~nm}$ in poly(3hexylthiophene) $(\mathrm{P} 3 \mathrm{HT})^{37,39}$ and likely also describes the absorption onset in P3MT CPB films.

The P3MT CPB films are especially notable in the appearance of a strongly blue-shifted absorption feature $\left(\lambda_{\max }\right.$ $\sim 415 \mathrm{~nm}$ in Figure $1 \mathrm{~b})^{22,23}$ with respect to the solution maxima of polymeric P3ATs. Regiorandom P3HT, with a persistence length (proxy for conjugation length) of $\sim 1 \mathrm{~nm}$, exhibits $\lambda_{\max } \sim 440 \mathrm{~nm}$, while regioregular $\mathrm{P} 3 \mathrm{HT}$, with a longer persistence length $(\sim 3 \mathrm{~nm})$, has a red-shifted $\lambda_{\max } \sim 470 \mathrm{~nm}^{40}$ As $\mathrm{H}$-aggregate coupling decreases with increasing conjugation length, the formation of $\mathrm{H}$-aggregates in the P3MT CPB films with absorption as at $415 \mathrm{~nm}$ implies that individual chromophores possess short/low conjugation units. This is supported by studies on the solution and film absorption behavior of oligothiophenes. Pentathiophene (5T) exhibits $\lambda_{\max }$ $\sim 420 \mathrm{~nm}$ in solution, while the nanoaggregate (H-type) has $\lambda_{\max } \sim 380 \mathrm{~nm}$, implying a $0.3 \mathrm{eV} \mathrm{H}$-aggregate coupling. ${ }^{41}$ The blue shift in the absorption of P3MT CPB films (i.e., $\sim 450$ to $415 \mathrm{~nm}, 0.25 \mathrm{eV})$ is consistent with the coupling observed in $5 \mathrm{~T}$, while the energy of the main feature in P3MT CPBs $(\sim 450$ $\mathrm{nm}$ ) implies a local conjugation length similar to that of regiorandom P3HT. Evidently, the coupling energy of $0.25 \mathrm{eV}$ greatly exceeds the common value of $0.1 \mathrm{eV}$ in P3HT films with long conjugation lengths.

The interpretation of the polarized, oblique incidence absorption spectra of P3MT CPB films requires numerical solutions of the anisotropic Fresnel equations (discussed further in the SI). Because of refraction, the electric field inside the film propagates significantly closer to the surface normal than the incident beam and thus under-samples the inplane component of the dielectric function. ${ }^{34,42,43}$ Because of interference effects, the $D R$ is thickness dependent for a given anisotropy. Here, we have modeled the thickness-dependent values of $D R$ for horizontal, isotropic and vertical anisotropy in very thin P3AT films, as shown in Figure 2a as colored lines (behavior determined from Figures S7 and S8). The thinnest P3MT CPB films (regime (i)), with $D R \sim 0.6$, are nominally isotropic $\left(\theta_{\text {iso }}=54.7^{\circ}\right)$, while the increase to $D R \sim 1.2$ for 30 $\mathrm{nm}$ thick films (regime (ii)) indicates a slight preference of the polymer chains toward vertical orientation $\left(\theta<\theta_{\text {iso }}\right)$. In the thickest films (regime (iii)), a $D R \sim 1$ suggests that the orientation of the polymer chains is nearly isotropic again. Oblique incidence $p$-polarized light exhibits resonances at the peak in $\operatorname{Im}[1 / \varepsilon]$ of the dielectric function $\varepsilon$ at the location of 
the longitudinal optical modes, i.e., the Berreman effect. ${ }^{44}$ This causes a significant blue shift in the $p$-polarized absorption with respect to the s-polarized counterpart for anisotropic films. The shift is absent in isotropic films. Thus, the spectra in Figure $2 b-$ $\mathrm{d}$ are consistent with the observed $D R$ : nominally isotropic in very thin and thick films, with a slight preference toward vertical anisotropy at intermediate $(\sim 30 \mathrm{~nm})$ thickness.

Analysis of Surface Structure by Atomic Force Microscopy (AFM). AFM topography images of P3MT CPB films have been analyzed in previous work, primarily for estimating film thickness and qualitative assessment of their surface structures. ${ }^{22-24}$ Here, the surface structure and morphology of the CPB films have been examined qualitatively and quantitatively using the AFM images, including the size and shape of surface features and their distributions. The surfaces of the P3MT CPB films consist of round columns with smooth and rounded tops, rising above a rough polymer network (Figure 3a). The heights of the columns vary significantly, with the tallest extending well over $100 \mathrm{~nm}$ above the surrounding area in the film. Scanning electron microscope images of the films are consistent with the formation of columns and an underlying polymer network (Figure S9). The column areal density, cross-sectional area, and their distribution were analyzed as a function of film thickness using the AFM images.

(a)
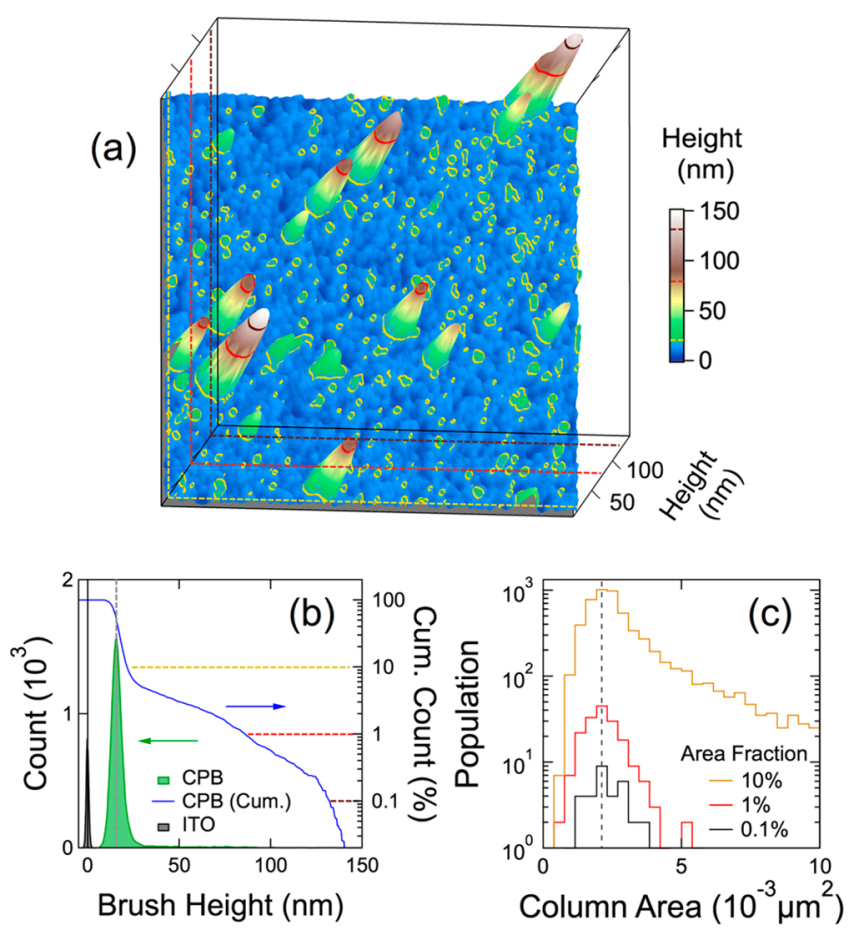

Figure 3. Typical AFM topography image of a $15 \mathrm{~nm}$ thick P3MT CPB film and its characteristic features. (a) 3D rendered AFM image. The yellow, red, and brown dashed lines and ribbons correspond to respective heights of 20,80 , and $130 \mathrm{~nm}$. The image dimensions in panel a are $3 \mu \mathrm{m} \times 3 \mu \mathrm{m}$, taken from a larger, $10 \mu \mathrm{m} \times 10 \mu \mathrm{m}$ AFM image used to produce panels $\mathrm{b}$ and $\mathrm{c}$. (b) Histogram (green) of the image in pixel count versus surface height and the corresponding normalized cumulative histogram (blue) from the highest point in the image (\% of pixels above a given height or area fraction). The horizontal dashed lines indicate the relationship between the heights and respective area fractions. Also shown is the histogram of the ITO surface exposed during scratch profilometry on the same film (black). (c) Distributions of cross-sectional area of columns at several area fractions.
The tall columns are of particular interest because they are the ones contacting the KTP printed top electrodes in CPB devices and thus primarily responsible for the charge transport processes (see below). The columns also provide insight into the growth mechanism of the P3MT CPB films. The growth mechanism and the polymer-electrode contacts are described in sections below.

The height histogram of each AFM image has a log-normal shape, and its corresponding cumulative histogram exhibits multiple exponential decays (Figure $3 \mathrm{~b}$ ). The full-width-at-halfmaximum (fwhm) of the histogram is very broad, about half of the film thickness, which is defined as the peak-to-peak distance between the histograms of the brush and the ITO surface (Figures $3 \mathrm{~b}$ and S2). To systematically probe the areal density and cross-sectional area of columns and their dependence on column height, these parameters were analyzed using several "threshold heights" for a given AFM image. For each threshold height, there is a corresponding threshold surface (i.e., the part of the surface above the height), which contains a certain fraction of the film surface area (i.e., pixels in the image), defined as the "area fraction" here. For example, for the $15 \mathrm{~nm}$ thick CPB film shown in Figure 3, 0.1\% of the surface area is taller than $130 \mathrm{~nm}$ (brown ribbons and lines), while 1\% (red ribbons and lines) and $10 \%$ (yellow ribbons and lines) of the areas are above respective heights of 80 and $20 \mathrm{~nm}$. Therefore, the threshold height that corresponds to a small area fraction is used to probe the parameters of tall columns, whereas the larger area fractions are used to analyze the short and the tall columns combined. Columns taller than a threshold height were counted to determine the areal density of columns above the threshold height, while the individual cross-sectional areas of the counted columns were measured to produce the area distribution of columns above the threshold height (Figure 3c). The cross-sectional area of columns was defined at a height $1 / 4$ of the film thickness below the highest point of the column. Other similar heights were used, and they do not qualitatively change the results of our analysis. The choice of this definition is primarily due to the convolution between the AFM tip and the columns, which causes increasing overestimation of the column cross-section area as the tip moves further down the sides of the columns (see SI for details).

The AFM topography measurements indicate that the columns form early in the film growth and exhibit a characteristic size and distribution. Specifically, taller and shorter columns have the same characteristic area. For the 15 $\mathrm{nm}$ thick film shown in Figure 3c, the peaks of the column area distributions at various area fractions are all about $2.2 \times 10^{-3}$ $\mu \mathrm{m}^{2}$ with fwhm values of $\sim 1 \times 10^{-3} \mu \mathrm{m}^{2}$. Analyses of 32 and 55 $\mathrm{nm}$ thick CPB films are also shown in Figures S10 and S11. Comparing between columns in films of different thicknesses, the characteristic column area and its distribution are nearly independent of the film thickness $(\geq 6 \mathrm{~nm})$, with an average value of $2.3 \pm 0.6 \times 10^{-3} \mu \mathrm{m}^{2}$ (Figure $4 \mathrm{a}$ ). In contrast, the surface height distributions scale with film thickness, such that the fwhm's of the height histograms are approximately half of the corresponding film thickness (Figure $4 b$ ).

The height-dependent column areal density exhibits a linear dependence on the area fraction over several orders of magnitude and is also independent of film thickness (open symbols in Figure 4c), consistent with the observation of a characteristic column area described above. The column density can be extrapolated to $100 \%$ area (i.e., the entire topography image) to yield a value of $200 \pm 50 \mu \mathrm{m}^{-2}$, corresponding to a 

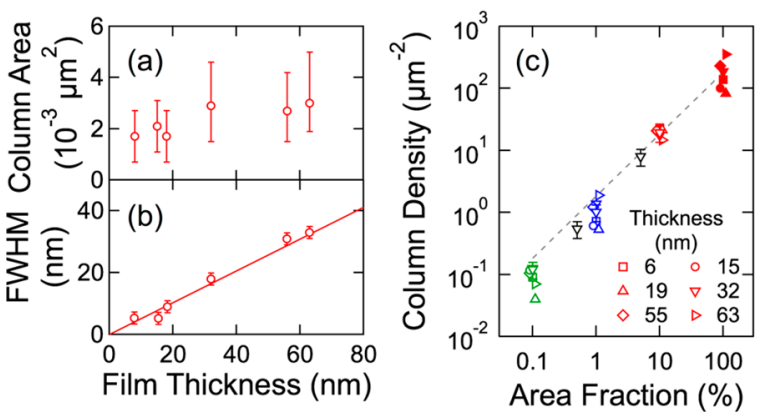

Figure 4. Characteristic surface and column parameters as a function of P3MT CPB film thickness. (a) Column cross-sectional. Error bars correspond to the width of the corresponding area distribution. (b) fwhm of the height histogram. The line is a linear fit with a slope of $0.51 \pm 0.02$. (c) Log plot of column density measured at various area fractions (open symbols) and that obtained from the height-height correlation function analysis (closed symbols). Films of different thicknesses are distinguished by symbols. The dashed line is a guide to the eyes to show linearity between the column density and the area fraction.

characteristic areal density of columns in the P3MT CPB films. Note that the spread of density values for films of different thicknesses at low area fractions $(\leq 1 \%)$ is likely the result of low sampling statistics of columns.

The height-height correlation function, $g(r)$, was used to further probe the characteristic length scales of the surface features in the AFM images of the P3MT CPB films. ${ }^{45,46}$ The $g(r)$ between two points on a surface separated by radius $r$ is given by

$$
\begin{aligned}
& g(r)=\left\langle\left[h(x, y)-h\left(x^{\prime}, y^{\prime}\right)\right]^{2}\right\rangle \text { and } \\
& r=\sqrt{\left(x-x^{\prime}\right)^{2}+\left(y-y^{\prime}\right)^{2}}
\end{aligned}
$$

where $h(x, y)$ is the height at position $(x, y)$. The $g(r)$ functions were first calculated from the AFM images and then fit using the phenomenological form

$$
g(r)=2 \sigma^{2}\left(1-e^{-(r / \xi)^{2 \alpha}}\right)
$$

where $\xi$ is the correlation length, $\alpha$ is the Hurst parameter (corresponding to a measure of short-range roughness), and $\sigma$ is the root-mean-square (RMS) roughness of the surface. This analysis produced thickness independent values for the correlation length and Hurst parameter, with respective values of $70 \pm 20 \mathrm{~nm}$ and $0.71 \pm 0.08$, and roughness values that depend linearly on film thickness (consistent with the result shown in Figure 4b). The correlation length directly probes the characteristic size or separation of surface features; ${ }^{46}$ as such, the inverse square of the correlation length corresponds to an areal density of surface features for the entire image. The densities from this analysis (closed symbols in Figure 4c) coincide with the extrapolated values from the column density analysis described above, indicating quantitative consistency between the two analyses. The value of the Hurst parameter (a measure of short-range surface roughness) corresponds to a relatively smooth surface on short length scales, also consistent with the observed smooth, rounded column tops. ${ }^{47}$ A typical height-height correlation function, corresponding fit, and parameters for films of various thicknesses are shown in Figure S12 and Table S1.

The agreement between the column analysis and heightheight correlation function analysis indicates that the parameters extracted by both originate from the same features on the surface of the P3MT CPB films. The columns therefore do not just account for the long tail in the height distribution of the films (e.g., Figure 3b) but instead comprise a significant portion of the surface area in P3MT CPB films. Therefore, it is reasonable to interpret that the height distribution of columns resembles the surface height distributions of the films, with column heights ranging from well above to below the nominal film thickness.

Summary of Morphological and Structural Characterization. On the basis of the observations made above using complementary techniques and analysis (primarily optical spectroscopy and AFM topography), a comprehensive picture of the structure and morphology of the P3MT CPB emerges. At the initial stage of growth ( $<10 \mathrm{~nm}$ thickness, regime (i)), polymer chains with moderate conjugation length (weak $\mathrm{H}$ aggregation with absorption onset at $\sim 600 \mathrm{~nm}$ ) and isotropic orientation are formed. There is an upward tilt on the local molecular scale between 10-30 nm (regime (ii)), coinciding with the emergence of self-supporting, round columns with a characteristic size and distribution. As chain growth continues (>30 nm thickness, regime (iii)), stronger $\mathrm{H}$-aggregates form between short conjugation units that may be correlated to the crystalline regions detected by GIWAXS. These aggregates dominate the absorption spectrum in thick films and have a nearly isotropic orientation. As the columns grow taller, their cross-sectional areas remain constant, while the column height distribution scales with film thickness. The observed tendency toward more isotropic orientation for films $>30 \mathrm{~nm}$ thickness may be the result of two factors: an aspect ratio of the columns greater than 1, giving rise to a tendency to form more tilted domains as they become less self-supporting, and desorption of the Pd catalyst into solution, reducing the number and density of propagating chains in thicker films. The latter is known to decrease vertical orientation in polymer brushes. ${ }^{48}$

Finally, the presence of a characteristic density and crosssectional area of the columns in the films is likely the result of the specific set of synthetic conditions used here. We expect that the characteristic P3MT CPB structures may be tunable by varying synthetic parameters, such as temperature, stirring rate, catalyst, monomer regioregularity and substitution, solvent and/or thermal annealing, and monolayer structure/density. The findings presented here therefore provide the means and impetus for future explorations into the interplay between the synthetic conditions and controlled structure and morphology in CPB films.

Polymer-Electrode Contact Analysis of CPB Devices. In most electrode-molecule-electrode devices, the contact between the electrodes and the active molecular layer is assumed to be conformal, and the distance between the top and bottom electrodes is assumed to be uniform, so the resistivity calculation is based entirely on the electrode area and the average molecular layer thickness. ${ }^{4,49,50}$ However, given the roughness of the P3MT CPB film surface, conformal contact between the film and top electrode is undesirable, as charge transport would be dominated by the thinnest parts of the device instead of the tall columns on the surface of the CPB films. To effectively study the charge transport through the P3MT CPB films, we employed the KTP process to print noncovalently attached $\mathrm{Au}$ electrodes onto the $\mathrm{CPB}$ films, producing arrays of ITO-CPB-Au trilayer devices (called CPB devices below) for $I-V$ measurements using conductive AFM (Figure S13). ${ }^{31}$ In order to calculate resistivity values of 
these devices, the contact between the CPBs and the fabricated $\mathrm{Au}$ electrodes as well as the CPB thickness within the devices must be examined. Because of the mechanical deformation of the Au electrode during the KTP process and the large widths present in the surface height distributions of the P3MT CPB films (Figures 3 and 4), the top $\mathrm{Au}$ electrode in the $\mathrm{CPB}$ devices presented here is neither conformal with the $\mathrm{CPB}$ surface nor at a uniform distance from the bottom electrode. To account for the two effects, a more general geometric contact factor is considered, in order to go beyond the widely used effective contact area. ${ }^{49,51-53}$

The shape of Au electrodes produced by photolithography is not a simple, flat disk, but instead the top surface is more like a dome with a rim (example shown in Figure 5a). The disks have

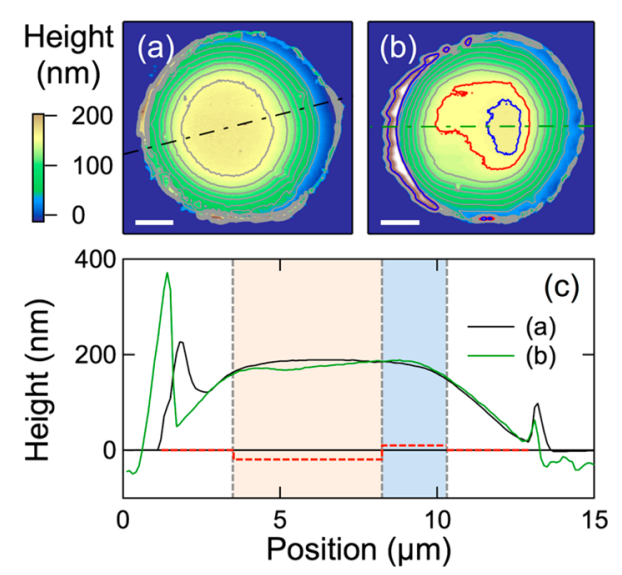

Figure 5. Representative AFM topography images of the KTP Au electrodes. (a) The undeformed, as-fabricated Au electrode on the donor Si substrate after HF etch and (b) a printed Au electrode on a $55 \mathrm{~nm}$ thick P3MT CPB film. In a and b, the horizontal scale bars correspond to $2 \mu \mathrm{m}$, and the gray contours are spaced at $20 \mathrm{~nm}$ intervals. (c) Comparison between linescans of the as-fabricated (black) and the printed (green) Au electrodes (positions of linescans indicated by dot-dashed lines in $\mathrm{a}$ and $\mathrm{b}$ ). Linescans are obtained along the high-symmetry axis of each pad. The zero height corresponds to the bottom of the as-fabricated electrode, while the red horizontal dashed lines approximate the bottom height of the deformed electrode. The blue and red contours in $\mathrm{b}$ correspond to deformed regions and to the light blue and pink shaded regions in c, respectively.

a relatively flat center, $185 \pm 5 \mathrm{~nm}$ thick, gradually decreasing radially to as thin as $15 \mathrm{~nm}$ around the edge (black trace in Figure 5c). The large difference in thickness between the edges and the center is evidently the result of a geometric shadowing effect between the photoresist pattern and the $\mathrm{Au}$ deposition angle. The bottoms of the as-fabricated $\mathrm{Au}$ electrodes are expected to be conformal to the donor $\mathrm{Si}$ substrates (as is typical with evaporated electrodes ${ }^{49}$ ) and thus flat.

The KTP process that involves lift off from the donor substrate and transfer to the $\mathrm{CPB}$ film causes the $\mathrm{Au}$ electrode to deform (Figure $5 \mathrm{~b}, \mathrm{c}$ ). These deformations are observed, to varying degrees, in $\sim 60 \%$ of electrodes printed on CPB films and can be characterized as primarily bending and buckling because other deformations of the Au electrode (in particular, a compressive change of thickness) would require a substantially greater force and stress than that used in KTP. This assumption that the KTP process does not cause a change in the thickness of the printed electrode is supported by our observation that none of the Au electrodes are deformed by KTP when printed onto flat Si substrates (Figure S14). Therefore, any deviations between the shape of the printed electrode surface (i.e., top of the electrode) and the as-fabricated counterpart caused by printing onto the rough CPB film are expected to be mirrored on the bottom of the printed electrode.

Because of the aforementioned deformation, the separation distance between the two electrodes in CPB devices varies from position to position (Figure 5c). To account for this variation, each deformed CPB device is divided into regions of parallel resistors, each with its own "regional" effective contact area $\left(A_{i}\right)$ and thickness (i.e., separation between electrodes, $L_{i}$ ). The "regional" effective contact area $A_{i}$ is generally smaller than the corresponding "regional" area of the electrode, owing to the surface roughness of the underlying $\mathrm{CPB}$ film, such that the total effective contact area of the device, $\sum_{i} A_{i}$, is far less than the area of the electrode, $A_{0}$.

For each device, the values of $L_{i}$ were estimated from the deformed bottom height of the printed electrode (Figure 5c) to the ITO surface. Tall columns in the CPB film reach heights above $L_{i}$ and thus make contact with the top electrode. Given that the elastic modulus of the Au electrode $(\sim 80 \mathrm{GPa})$ is likely to be much larger than that of P3MT (the elastic modulus of P3HT is $\sim 1 \mathrm{GPa}),{ }^{54,55}$ we assume that the columns above $L_{i}$ compress down to the bottom of the Au electrode, while compression of the Au electrode is negligible. This assumption serves as an upper bound for the amount of contact between the $\mathrm{CPB}$ film and $\mathrm{Au}$ electrode, as any compression of the $\mathrm{Au}$ electrode that is ignored here would cause an increase in $L_{i}$ and a decrease in the contact factor below. Because only the tallest portion of the CPB films are in contact with the Au electrode, values of $L_{i}$ are much greater than the nominal film thickness, $L_{0}$. These tall columns represent a small fraction of the film surface area, causing the "regional" effective contact area $A_{i}$ to depend very sensitively on $L_{i}$. The surface height histogram and the corresponding cumulative histogram were used to determine $A_{i}$ for a given $L_{i}$ (Figure 6). Specifically, $A_{i}$ is the area fraction of the CPB film (horizontal dashed lines) that is above $L_{i}$ (vertical dashed lines).

Assuming that there is uniform resistivity $(\rho)$ across all regions within each device, the resistance $(R)$ consisting of parallel resistors is given by

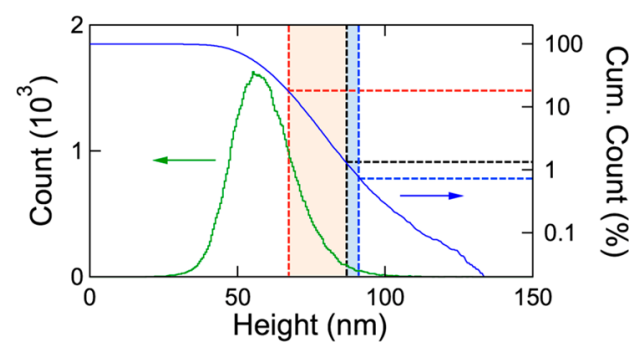

Figure 6. Typical surface height histogram (green) and corresponding normalized cumulative histogram (blue, cumulative from the highest point of the image) of a $55 \mathrm{~nm}$ thick P3MT CPB film and intersection with a deformed, printed Au electrode (shown in Figure $5 \mathrm{~b}$ and $\mathrm{c}$ ). The zero height is at the surface of the ITO substrate. Vertical dashed lines correspond to the estimated "regional" heights of the bottom of the top electrode ( $L_{i}$ in the text). The horizontal dashed lines indicate the respective area fractions of the CPB film making contact with the top electrode ( $A_{i}$ in the text). The blue and red dashed lines correspond to the contacts of the deformed parts of the electrode from Figure $5 \mathrm{~b}$ and $\mathrm{c}$, whereas the black dashed lines correspond to that of the undeformed part. 


$$
\begin{aligned}
& R=\rho \frac{L_{0}}{A_{0} \cdot a} \text { and } \\
& a=\sum_{i} \frac{A_{i} / A_{0}}{L_{i} / L_{0}}
\end{aligned}
$$

where $a$ is the effective contact factor introduced as a correction to the nominal resistivity of the device. Our analysis of many samples and devices indicates that the average value for the effective contact area (i.e., $\left(\sum_{i} A_{i} / A_{0}\right)$ is $2 \%$ and is independent of the CPB film thickness. The effective contact factor $a$ is also independent of film thickness, with a log average value of -2.0 \pm 0.6 , corresponding to an average value of $1 \%$ with lower and upper bounds of $0.25 \%$ and $4 \%$ (Table S2). These findings are likely the result of the observed film thickness independent column density and cross-sectional area and the resulting mechanical properties of the columns, combined with the consistency of the KTP process.

Characterization of Charge Transport through CPB Devices. The $I-V$ curves of the P3MT CPB devices show the characteristic nonlinear behavior typical of narrow gap semiconductors (Figure S15). The zero-bias resistance of the devices exhibits linear behavior with respect to the CPB film thickness, consistent with diffusive transport processes outside of the tunneling regime (Figure 7 ). ${ }^{18} \mathrm{~A}$ bulk resistivity value

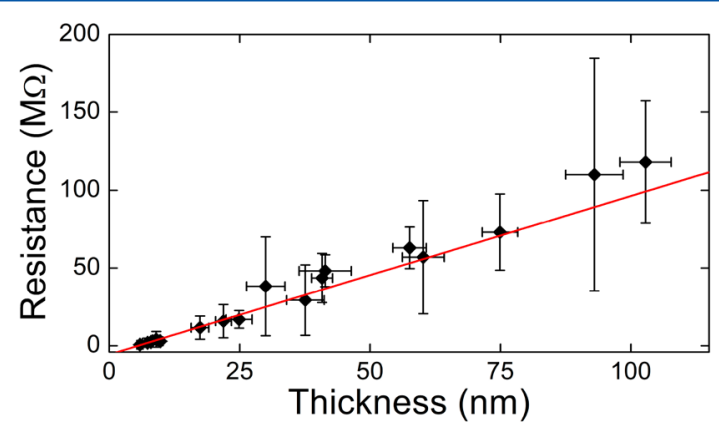

Figure 7. Zero-bias resistance vs film thickness of P3MT CPB devices. Each point corresponds to measurements on more than 20 devices. The red line is a linear fit with a slope of $1.0 \pm 0.1 \mathrm{M} \Omega / \mathrm{nm}$. Note that the nonzero intercept is typical for molecular wires. ${ }^{60}$

along the undoped P3MT CPB columns of $1.4 \times 10^{5} \Omega \cdot \mathrm{cm}$ (lower and upper bounds of 0.4 and $5.6 \times 10^{5} \Omega \cdot \mathrm{cm}$, respectively) was calculated from eqs 4 and 5 using $\rho / L_{0}=1.0$ $\mathrm{M} \Omega / \mathrm{nm}$ (slope of Figure 7), $A_{0}=120 \mu \mathrm{m}^{2}$, and $a=1 \%$. This value is 2 orders of magnitude lower than the typical room temperature values for spuncast, undoped P3HT films measured using 4-point probe and Van der Pauw techniques. However, these in-plane, 4-terminal techniques are not compatible for studying vertical intramolecular transport through P3MT CPBs. ${ }^{56,57}$ Note that since resistivity of P3MT CPB devices is determined from the slope of the resistance versus thickness plot, contact resistance and other interfacial effects (e.g., the size of the injection barrier at the interface) are excluded.

Values for the drift mobility in the P3MT CPBs cannot be obtained from $I-V$ behavior using analytical models (e.g., space-charge or injection limited current) owing to non-Ohmic contacts between the two electrodes and P3MT molecules and the presence of a large interfacial barrier introduced by the phosphonic acid monolayer. However, the intrinsic carrier concentration in poly(3-alkylthiophene)s has a weak depend- ence on the side chain structure, ${ }^{58,59}$ so the observed large reduction in the bulk resistivity of $\mathrm{P} 3 \mathrm{MT}$ CPBs provides evidence for a corresponding enhancement of mobility in the P3MT CPB films compared to spuncast P3HT. Furthermore, improvements on the vertical chain alignment of P3MT chains through efforts to optimize synthetic parameters may lead to even higher values of mobility in these films.

To compare the conduction along P3MT CPBs with single molecule/ensemble devices in which intramolecular hopping has been reported to dominate charge transport processes, the average resistance along individual polymer chains as a function of length (i.e., molecular resistivity) was estimated. For the P3MT CPB films, the molecular density inside the tall column domains $\left(N_{c}\right)$ was calculated to have an upper bound of 2.6 $\mathrm{nm}^{-2}$ (see SI for details) and used to convert the bulk resistivity value to a molecular value of $360 \mathrm{G} \Omega / \mathrm{nm}$ (with lower and upper bounds of 90 and $1400 \mathrm{G} \Omega / \mathrm{nm}$, respectively) per P3MT molecule. We note that this estimate assumes that the polymer chain density stays constant during film growth and that the device thickness is equal to the polymer chain length, both of which would overestimate the value for molecular resistivity. Although neither factor can be quantified at this time, $\mathrm{Pd}$ desorption during polymerization likely causes a reduction in chain density as the film grows (resulting in a lower $N_{c}$ ). The polymer chains in these films also likely undergo significant twisting and winding between the bottom and top electrodes, as they are significantly tilted and disordered (from the spectroscopy experiments described above), so the polymer chain lengths within the columns are evidently much longer than the device thickness (the molecular weight of the P3MT polymer cannot be directly measured owing to its insolubility). Therefore, the estimated molecular resistivity value for P3MT should be considered as an upper bound. However, this value is already comparable to short, conjugated molecular wires that exhibit predominantly intramolecular charge transport, e.g., 340 $\mathrm{G} \Omega / \mathrm{nm}$ for the conjugated oligophenyleneimine molecular wires synthesized by Choi et al. (see SI for calculation). ${ }^{18,19,21,61-64}$

The observed reduction in the bulk resistivity compared to that of spuncast counterparts and the low molecular resistivity value in the P3MT CPB devices support the notion that intramolecular charge transport processes are responsible for enhancing charge conduction through these films. The primary enabling factor is that all of the P3MT molecules in contact with the top Au electrode are covalently bound to the bottom ITO electrode. We do expect that intermolecular processes contribute to conduction given the presence of extensive intermolecular interactions, as indicated by the optical spectroscopy results. However, the extent of the intermolecular contribution cannot currently be determined. Finally, investigations to date have focused on characterizing intramolecular charge transport processes in molecular wires less than $10 \mathrm{~nm}$ long and in devices containing $<100$ molecules (often single molecules). This work on P3MT CPB devices has incorporated orders of magnitude more molecular wires reaching lengths well over $100 \mathrm{~nm}$, demonstrating that the CPB devices constitute a novel and scalable platform for studying intramolecular transport phenomena.

\section{CONCLUSIONS}

In summary, we report the morphological, structural, and electrical characteristics of P3MT conjugated polymer brushes (CPBs). The growth of the polymer chains in these films 
exhibits three distinct regimes: the orientation of the polymer chains is nearly isotropic in films thinner than $10 \mathrm{~nm}$ and thicker than $30 \mathrm{~nm}$, while it becomes slightly vertical in between. Early in film growth, the formation of columns is observed on the film surface. Analysis of the columns indicates that they exhibit a characteristic size and density that is independent of film thickness, whereas the column height distribution scales with film thickness. We expect that the column characteristics may be tuned by changing various synthetic parameters during polymerization.

Electrical conduction through the columns was studied in the electrode-CPB-electrode devices with $\mathrm{Au}$ electrodes printed on top of the CPB films. Analysis of the interface between the $\mathrm{Au}$ electrode and the $\mathrm{CPB}$ film was performed in order to address the deformation of the $\mathrm{Au}$ electrodes during device fabrication. After correcting for these effects by using a contact factor, the bulk resistivity along the columns was determined to be 100 times lower than the typical values for spuncast, poly(3alkylthiophene) films. Furthermore, the resistance along individual P3MT polymer chains in the conjugated polymer brush films was determined to be comparable to the literature values for very short $(<10 \mathrm{~nm})$ molecular wires. These findings strongly suggest the presence of enhanced intramolecular conduction along P3MT polymer chains, thus making conjugated polymer brush films a promising and unexplored platform to study the interplay among the synthetic conditions, film morphology, and structure, and intramolecular charge transport phenomena in organic semiconductors.

\section{ASSOCIATED CONTENT}

\section{S Supporting Information}

The Supporting Information is available free of charge on the ACS Publications website at DOI: 10.1021/acs.jpcc.8b00033.

Additional figures related to polarized UV-vis spectroscopy, scratch profilometry, cyclic voltammetry, X-ray scattering, simulated absorption spectra of P3HT films, scanning electron microscopy images, atomic force microscopy images, height-height correlation function analysis, microscopy of $\mathrm{Au}$ electrodes, $\mathrm{I}-V$ curves, and additional information on optical modeling and column analysis (PDF).

\section{AUTHOR INFORMATION}

\section{Corresponding Authors}

*(F.T.) E-mail: ftsui@physics.unc.edu.

*(W.Y.) E-mail: wyou@unc.edu.

\section{ORCID}

Ian A. VonWald: 0000-0001-9575-1840

Jason Locklin: 0000-0001-9272-2403

Wei You: 0000-0003-0354-1948

\section{Notes}

Certain commercial equipment, instruments, or materials are identified in this article in order to specify the experimental procedure adequately. Such identification is not intended to imply recommendation or endorsement by the National Institute of Standards and Technology, nor is it intended to imply that the materials or equipment identified are necessarily the best available for the purpose.

The authors declare no competing financial interest.

\section{ACKNOWLEDGMENTS}

We acknowledge the following support for this collaborative research: I.V.W. was supported by the National Science Foundation Graduate Research Fellowship under Grant No. DGE-1650116. J.Y. and W.Y. were supported by NSF Grants No. CHE-1412286 and No. DMR-1610879. J.L. was supported by NSF Grant No. CHE-1412714. Parts of this work were performed in part at the Chapel Hill Analytical and Nanofabrication Laboratory, CHANL, a member of the North Carolina Research Triangle Nanotechnology Network, RTNN, which is supported by the National Science Foundation, Grant ECCS-1542015, as part of the National Nanotechnology Coordinated Infrastructure, NNCI. Beamline 7.3.3 of the Advanced Light Source is supported by the Director of the Office of Science, Office of Basic Energy Sciences, of the U.S. Department of Energy under Contract No. DE-AC02-05CH11231. The authors thank Lee J. Richter for performing GIWAXS and for discussions on the interpretation of spectroscopic data. We thank Brett Bowman for the synthesis of the P3MT monomer.

\section{REFERENCES}

(1) Coropceanu, V.; Cornil, J.; da Silva Filho, D. A.; Olivier, Y.; Silbey, R; Brédas, J. L. Charge Transport in Organic Semiconductors. Chem. Rev. 2007, 107, 926-952.

(2) Noriega, R.; Rivnay, J.; Vandewal, K.; Koch, F. P. V; Stingelin, N.; Smith, P.; Toney, M. F.; Salleo, A. A General Relationship between Disorder, Aggregation and Charge Transport in Conjugated Polymers. Nat. Mater. 2013, 12, 1038-1044.

(3) Zhang, X.; Bronstein, H.; Kronemeijer, A. J.; Smith, J.; Kim, Y.; Kline, R. J.; Richter, L. J.; Anthopoulos, T. D.; Sirringhaus, H.; Song, K.; et al. Molecular Origin of High Field-Effect Mobility in an Indacenodithiophene-Benzothiadiazole Copolymer. Nat. Commun. 2013, 4, 2238.

(4) Skrypnychuk, V.; Wetzelaer, G. J. A. H.; Gordiichuk, P. I.; Mannsfeld, S. C. B.; Herrmann, A.; Toney, M. F.; Barbero, D. R. Ultrahigh Mobility in an Organic Semiconductor by Vertical Chain Alignment. Adv. Mater. 2016, 28, 2359-2366.

(5) O'Connor, B.; Kline, R. J.; Conrad, B. R.; Richter, L. J.; Gundlach, D.; Toney, M. F.; DeLongchamp, D. M. Anisotropic Structure and Charge Transport in Highly Strain-Aligned Regioregular Poly(3Hexylthiophene). Adv. Funct. Mater. 2011, 21, 3697-3705.

(6) Grozema, F. C.; Siebbeles, L. D. A. Charge Mobilities in Conjugated Polymers Measured by Pulse Radiolysis Time-Resolved Microwave Conductivity: From Single Chains to Solids. J. Phys. Chem. Lett. 2011, 2, 2951-2958.

(7) Lan, Y.-K.; Yang, C. H.; Yang, H.-C. Theoretical Investigations of Electronic Structure and Charge Transport Properties in Polythiophene-Based Organic Field-Effect Transistors. Polym. Int. 2010, 59, $16-21$.

(8) Soo Yook, K.; Doo Chin, B.; Yeob Lee, J.; Lassiter, B. E.; Forrest, S. R. Vertical Orientation of Copper Phthalocyanine in Organic Solar Cells Using a Small Molecular Weight Organic Templating Layer. Appl. Phys. Lett. 2011, 99, 043308.

(9) Yang, Y.; Mielczarek, K.; Aryal, M.; Zakhidov, A.; Hu, W. Effects of Nanostructure Geometry on Nanoimprinted Polymer Photovoltaics. Nanoscale 2014, 6, 7576-7584.

(10) Venkataraman, D.; Yurt, S.; Venkatraman, B. H.; Gavvalapalli, N. Role of Molecular Architecture in Organic Photovoltaic Cells. J. Phys. Chem. Lett. 2010, 1, 947-958.

(11) Watanabe, Y.; Sasabe, H.; Yokoyama, D.; Beppu, T.; Katagiri, H.; Pu, Y.-J.; Kido, J. Simultaneous Manipulation of Intramolecular and Intermolecular Hydrogen Bonds in N-Type Organic Semiconductor Layers: Realization of Horizontal Orientation in OLEDs. Adv. Opt. Mater. 2015, 3, 769-773. 
(12) Trivedi, K.; Bhansali, U. S.; Gnade, B.; Hu, W. The Fabrication of High Density Nanochannel Organic Light Emitting Diodes with Reduced Charge Spreading. Nanotechnology 2009, 20, 405204.

(13) Menard, E.; Meitl, M. A.; Sun, Y.; Park, J.-U.; Shir, D. J.-L.; Nam, Y.-S.; Jeon, S.; Rogers, J. A. Micro- and Nanopatterning Techniques for Organic Electronic and Optoelectronic Systems. Chem. Rev. 2007, 107, 1117-1160.

(14) Park, S.; Lim, B. T.; Kim, B.; Son, H. J.; Chung, D. S. High Mobility Polymer Based on a $\pi$-Extended Benzodithiophene and Its Application for Fast Switching Transistor and High Gain Photoconductor. Sci. Rep. 2015, 4, 5482.

(15) Mas-Torrent, M.; Rovira, C. Role of Molecular Order and SolidState Structure in Organic Field-Effect Transistors. Chem. Rev. 2011, 111, 4833-4856.

(16) Li, F.; Li, T.; Chen, F.; Zhang, F. Excellent Spin Transport in Spin Valves Based on the Conjugated Polymer with High Carrier Mobility. Sci. Rep. 2015, 5, 9355.

(17) Ma, J.; Hashimoto, K.; Koganezawa, T.; Tajima, K. End-On Orientation of Semiconducting Polymers in Thin Films Induced by Surface Segregation of Fluoroalkyl Chains. J. Am. Chem. Soc. 2013, 135, 9644-9647.

(18) Ho Choi, S.; Kim, B.; Frisbie, C. D. Electrical Resistance of Long Conjugated Molecular Wires. Science 2008, 320, 1482-1486.

(19) Smith, C. E.; Odoh, S. O.; Ghosh, S.; Gagliardi, L.; Cramer, C. J.; Frisbie, C. D. Length-Dependent Nanotransport and Charge Hopping Bottlenecks in Long Thiophene-Containing $\pi$-Conjugated Molecular Wires. J. Am. Chem. Soc. 2015, 137, 15732-15741.

(20) Sangeeth, C. S. S.; Demissie, A. T.; Yuan, L.; Wang, T.; Frisbie, C. D.; Nijhuis, C. A. Comparison of DC and AC Transport in 1.5-7.5 $\mathrm{nm}$ Oligophenylene Imine Molecular Wires across Two Junction Platforms: Eutectic Ga-In versus Conducting Probe Atomic Force Microscope Junctions. J. Am. Chem. Soc. 2016, 138, 7305-7314.

(21) Lafferentz, L.; Ample, F.; Yu, H.; Hecht, S.; Joachim, C.; Grill, L. Conductance of a Single Conjugated Polymer as a Continuous Function of Its Length. Science 2009, 323, 1193-1197.

(22) Huddleston, N. E.; Sontag, S. K.; Bilbrey, J. A.; Sheppard, G. R.; Locklin, J. Palladium-Mediated Surface-Initiated Kumada Catalyst Polycondensation: A Facile Route Towards Oriented Conjugated Polymers. Macromol. Rapid Commun. 2012, 33, 2115-2120.

(23) Doubina, N.; Jenkins, J. L.; Paniagua, S. A.; Mazzio, K. A.; MacDonald, G. A.; Jen, A. K. Y.; Armstrong, N. R.; Marder, S. R.; Luscombe, C. K. Surface-Initiated Synthesis of poly(3-Methylthiophene) from Indium Tin Oxide and Its Electrochemical Properties. Langmuir 2012, 28, 1900-1908.

(24) Youm, S. G.; Hwang, E.; Chavez, C. A.; Li, X.; Chatterjee, S.; Lusker, K. L.; Lu, L.; Strzalka, J.; Ankner, J. F.; Losovyj, Y.; et al. Polythiophene Thin Films by Surface-Initiated Polymerization: Mechanistic and Structural Studies. Chem. Mater. 2016, 28, 47874804.

(25) Senkovskyy, V.; Khanduyeva, N.; Komber, H.; Oertel, U.; Stamm, M.; Kuckling, D.; Kiriy, A. Conductive Polymer Brushes of Regioregular Head-to-Tail poly(3-Alkylthiophenes) via CatalystTransfer Surface-Initiated Polycondensation. J. Am. Chem. Soc. 2007, $129,6626-6632$.

(26) Sontag, S. K.; Sheppard, G. R.; Usselman, N. M.; Marshall, N.; Locklin, J. Surface-Confined Nickel Mediated Cross-Coupling Reactions: Characterization of Initiator Environment in Kumada Catalyst-Transfer Polycondensation. Langmuir 2011, 27, 1203312041 .

(27) Roy, A.; Bougher, T. L.; Geng, R.; Ke, Y.; Locklin, J.; Cola, B. A. Thermal Conductance of Poly(3-Methylthiophene) Brushes. ACS Appl. Mater. Interfaces 2016, 8, 25578-25585.

(28) Geng, R.; Roy, A.; Zhao, W.; Subedi, R. C.; Li, X.; Locklin, J.; Nguyen, T. D. Engineering of Spin Injection and Spin Transport in Organic Spin Valves Using $\pi$-Conjugated Polymer Brushes. Adv. Funct. Mater. 2016, 26, 3999-4006.

(29) Yang, L.; Sontag, S. K.; LaJoie, T. W.; Li, W.; Huddleston, N. E.; Locklin, J.; You, W. Surface-Initiated Poly(3-Methylthiophene) as a
Hole-Transport Layer for Polymer Solar Cells with High Performance. ACS Appl. Mater. Interfaces 2012, 4, 5069-5073.

(30) LaJoie, T. W. Conjugated Polymer Brushes as Molecular Wires in Electronic Devices: Synthesis, Device Design, and Characterization. $\mathrm{Ph}$. D. Dissertation, University of North Carolina at Chapel Hill, 2015.

(31) Meitl, M. a.; Zhu, Z.-T.; Kumar, V.; Lee, K. J.; Feng, X.; Huang, Y. Y.; Adesida, I.; Nuzzo, R. G.; Rogers, J. A. Transfer Printing by Kinetic Control of Adhesion to an Elastomeric Stamp. Nat. Mater. 2006, 5, 33-38.

(32) Ilavsky, J. Nika: Software for Two-Dimensional Data Reduction. J. Appl. Crystallogr. 2012, 45, 324-328.

(33) Bruce, R. C.; Wang, R.; Rawson, J.; Therien, M. J.; You, W. Valence Band Dependent Charge Transport in Bulk Molecular Electronic Devices Incorporating Highly Conjugated Multi[(Porphinato)Metal] Oligomers. J. Am. Chem. Soc. 2016, 138, 2078-2081.

(34) Gurau, M. C.; Delongchamp, D. M.; Vogel, B. M.; Lin, E. K.; Fischer, D. A.; Sambasivan, S.; Richter, L. J. Measuring Molecular Order in Poly(3-Alkylthiophene) Thin Films with Polarizing Spectroscopies. Langmuir 2007, 23, 834-842.

(35) Yamamoto, T.; Komarudin, D.; Arai, M.; Lee, B.-L.; Suganuma, H.; Asakawa, N.; Inoue, Y.; Kubota, K.; Sasaki, S.; Fukuda, T.; et al. Extensive Studies on $\pi$-Stacking of Poly(3-Alkylthiophene-2,5-Diyl)s and Poly(4-Alkylthiazole-2,5-Diyl)s by Optical Spectroscopy, NMR Analysis, Light Scattering Analysis, and X-Ray Crystallography. J. Am. Chem. Soc. 1998, 120, 2047-2058.

(36) Yamagata, H.; Spano, F. C. Interplay between Intrachain and Interchain Interactions in Semiconducting Polymer Assemblies: The HJ-Aggregate Model. J. Chem. Phys. 2012, 136, 184901.

(37) Spano, F. C. Modeling Disorder in Polymer Aggregates: The Optical Spectroscopy of Regioregular Poly(3-Hexylthiophene) Thin Films. J. Chem. Phys. 2005, 122, 234701.

(38) Gierschner, J.; Huang, Y.-S.; Van Averbeke, B.; Cornil, J.; Friend, R. H.; Beljonne, D. Excitonic versus Electronic Couplings in Molecular Assemblies: The Importance of Non-Nearest Neighbor Interactions. J. Chem. Phys. 2009, 130, 044105.

(39) Clark, J.; Chang, J.-F.; Spano, F. C.; Friend, R. H.; Silva, C. Determining Exciton Bandwidth and Film Microstructure in Polythiophene Films Using Linear Absorption Spectroscopy. Appl. Phys. Lett. 2009, 94, 163306

(40) McCulloch, B.; Ho, V.; Hoarfrost, M.; Stanley, C.; Do, C.; Heller, W. T.; Segalman, R. A. Polymer Chain Shape of poly(3Alkylthiophenes) in Solution Using Small-Angle Neutron Scattering. Macromolecules 2013, 46, 1899-1907.

(41) Egelhaaf, H.-J.; Gierschner, J.; Haiber, J.; Oelkrug, D. Optical Constants of Highly Oriented Oligothiophene Films and Nanoparticles. Opt. Mater. 1999, 12, 395-401.

(42) Wang, F.; Hashimoto, K.; Tajima, K. Optical Anisotropy and Strong H-Aggregation of Poly(3-Alkylthiophene) in a Surface Monolayer. Adv. Mater. 2015, 27, 6014-6020.

(43) Tomlin, S. G. Optical Reflection and Transmission Formulae for Thin Films. J. Phys. D: Appl. Phys. 1968, 1, 1667.

(44) Harbecke, B.; Heinz, B.; Grosse, P. Optical Properties of Thin Films and the Berreman Effect. Appl. Phys. A. 1985, 38, 263-267.

(45) Sinha, S. K.; Sirota, E. B.; Garoff, S.; Stanley, H. B. X-Ray and Neutron Scattering from Rough Surfaces. Phys. Rev. B. 1988, 38, 2297-2311.

(46) Stone, V.; Jonas, A. M.; Nysten, B.; Legras, R. Roughness of Free Surfaces of Bulk Amorphous Polymers as Studied by X-Ray Surface Scattering and Atomic Force Microscopy. Phys. Rev. B. 1999, $60,5883-5894$.

(47) Krim, J.; Indekeu, J. O. Roughness Exponent: A Paradox Resolved. Phys. Rev. E. 1993, 48, 1576-1578.

(48) Wu, T.; Efimenko, K.; Genzer, J. Combinatorial Study of the Mushroom-to-Brush Crossover in Surface Anchored Polyacrylamide. J. Am. Chem. Soc. 2002, 124, 9394-9395.

(49) Simeone, F. C.; Yoon, H. J.; Thuo, M. M.; Barber, J. R.; Smith, B.; Whitesides, G. M. Defining the Value of Injection Current and 
Effective Electrical Contact Area for EGaIn-Based Molecular Tunneling Junctions. J. Am. Chem. Soc. 2013, 135, 18131-18144.

(50) Jeong, H.; Kim, D.; Xiang, D.; Lee, T. High-Yield Functional Molecular Electronic Devices. ACS Nano 2017, 11, 6511-6548.

(51) Merces, L.; de Oliveira, R. F.; de Camargo, D. H. S.; Bufon, C.

C. B. Long-Range Coherent Tunneling in Physisorbed Molecular Ensembles. J. Phys. Chem. C 2017, 121, 16673-16681.

(52) Niskala, J. R.; You, W. Metal-Molecule-Metal Junctions via PFPE Assisted Nanotransfer Printing (nTP) onto Self-Assembled Monolayers. J. Am. Chem. Soc. 2009, 131, 13202-13203.

(53) Niskala, J. R.; Rice, W. C.; Bruce, R. C.; Merkel, T. J.; Tsui, F.; You, W. Tunneling Characteristics of Au-Alkanedithiol-Au Junctions Formed via Nanotransfer Printing (nTP). J. Am. Chem. Soc. 2012, 134, 12072-12082.

(54) Volinsky, A. A.; Moody, N. R.; Gerberich, W. W. Nanoindentation of $\mathrm{Au}$ and $\mathrm{Pt} / \mathrm{Cu}$ Thin Films at Elevated Temperatures. J. Mater. Res. 2004, 19, 2650-2657.

(55) Awartani, O.; Lemanski, B. I.; Ro, H. W.; Richter, L. J.; DeLongchamp, D. M.; O'Connor, B. T. Correlating Stiffness, Ductility, and Morphology of Polymer:Fullerene Films for Solar Cell Applications. Adv. Energy Mater. 2013, 3, 399-406.

(56) Foot, P.; Swiatek, J.; Szymanska, T. Conductivity of Poly(3Hexathiophene) and Its Application to Electrical Devices. Mol. Cryst. Liq. Cryst. Sci. Technol., Sect. A 1993, 229, 225-228.

(57) Caruso, A. N.; Feng, D.-Q.; Losovyj, Y. B.; Schulz, D. L.; Balaz, S.; Rosa, L. G.; Sokolov, A.; Doudin, B.; Dowben, P. A. Defect Contributions to Conductivity in poly(3-Hexylthiophene)? Phys. Status Solidi B 2006, 243, 1321-1330.

(58) Kim, F. S.; Jenekhe, S. A. Charge Transport in Poly(3Butylthiophene) Nanowires and Their Nanocomposites with an Insulating Polymer. Macromolecules 2012, 45, 7514-7519.

(59) Ecker, B.; Nolasco, J. C.; Pallarés, J.; Marsal, L. F.; Posdorfer, J.; Parisi, J.; Von Hauff, E. Degradation Effects Related to the Hole Transport Layer in Organic Solar Cells. Adv. Funct. Mater. 2011, 21, 2705-2711.

(60) Luo, L.; Choi, S. H.; Frisbie, C. D. Probing Hopping Conduction in Conjugated Molecular Wires Connected to Metal Electrodes. Chem. Mater. 2011, 23, 631-645.

(61) Choi, S. H.; Risko, C.; Delgado, M. C. R.; Kim, B.; Brédas, J. L.; Frisbie, C. D. Transition from Tunneling to Hopping Transport in Long, Conjugated Oligo-Imine Wires Connected to Metals. J. Am. Chem. Soc. 2010, 132, 4358-4368.

(62) Luo, L.; Frisbie, C. D. Length-Dependent Conductance of Conjugated Molecular Wires Synthesized by Stepwise "Click" Chemistry. J. Am. Chem. Soc. 2010, 132, 8854-8855.

(63) Yamada, R.; Kumazawa, H.; Tanaka, S.; Tada, H. Electrical Resistance of Long Oligothiophene Molecules. Appl. Phys. Express 2009, 2, 025002.

(64) Lu, Q.; Liu, K.; Zhang, H.; Du, Z.; Wang, X.; Wang, F. From Tunneling to Hopping: A Comprehensive Investigation of Charge Transport Mechanism in Molecular Junctions Based on Oligo(pPhenylene Ethynylene)s. ACS Nano 2009, 3, 3861-3868. 